\section{SAT0022 THE SUBPOPULATION FEATURES OF DENDRITIC CELLS AS A POTENTIAL BIOMARKER OF EARLY RHEUMATOID ARTHRITIS}

Maxim Korolev, Yuliya Kurochkina, Nadezda Banshhikova, Vitaly Omelchenko, Anna Akimova, Elena Letyagina, Mariya Surovtseva, Alexander Lykov, Olga Poveschenko. Research Institute of Clinical and Experimental Lymphology branch of the Institute of Cytology and Genetics, Siberian Branch of the Russian Academy of Sciences, 2, Tymakova street, Novosibirsk, Russian Federation

Background: Dendritic cells (DCs) are professional antigen-presenting cells (APCs), which play important role in immune responses. DCs are a heterogeneous population and can be divided into groups: myeloid (mDCs) and plasmacytoid (pDCs). Furthemore, DCs are important in rheumatoid arthritis (RA) pathogenesis through antigen presentation and activation autoreactive T-lymphocytes. As established the different subpopulation DCs can promote different immune reactions. That's why, it may be possible to consider them as a potential target for the development of new target of the immunopathological disorders therapy.

Objectives: To investigate the subpopulations of peripheral blood DCs (myeloid and plasmacytoid) in patients with early RA as a predictor of responsibility to disease-modifying antirheumatic drugs (DMARDs) treatment.

Methods: Forty nine patients with early RA (duration of the disease up to 12 months) were included in the study. All patients fullfield ACR/ EULAR criteria (2010) and received methotrexate, leflunomide, sulfasalazine or their combination. Forty three patients with osteoarthritis (OA) used as a control group. Analysis of the content of the B-lymphocytes, myeloid and plasmacytoid DCs, labeled by antibodies against surface markers, was carried out by flow cytometry. B-lymphocytes, subtypes of peripheral blood DCs were characterized by the following phenotypes: myeloid DCs (CD3-CD14-CD19-HLA-DR + CD11C + CD123-), plasmacytoid DCs (CD3-CD14-CD19-HLA-DR + CD11C-CD123 +), B-lymphocytes $($ CD19 +). Analysis were performed before treatment and after 3 and 6 months.

Results: Patients with early RA are characterized by significant evaluation of the population of plasmacytoid DCs in comparison of patients with moderate stages of rheumatoid arthritis and osteoarthritis (3.8 vs2.1 vs 1 , $\mathrm{p}=0.0042$ ). Furthermore, the difference was found in the number of cells with the phenotype B-lymphocytes: $7.95 * 106 / 1$ vs. $3.6^{*} 106 /$, respectively $(p=0.014)$. No significant differences were observed in the number of myeloid DCs. After 6 month of observation we detected reducing amount of plasmacytoid DCs (3.8 before treatment and 2.1 in 6 month, $\mathrm{p}=0.002$ ) and B-cells that correlated with activity of disease.

Conclusion: The data obtained indicated that plasmacytoid DCs are predominant in patients with inflammatory arthritis especially in early RA and correlate with activity of disease that can use as a predictor of good response on DMARDs treatment.

Disclosure of Interests: None declared

DOI: 10.1136/annrheumdis-2019-eular.6783

\section{SAT0023 THE DIFFERENTIAL PRODUCTION OF REACTIVE OXYGEN SPECIES IN T CELL SUBSETS IN PERIPHERAL BLOOD OF RHEUMATOID ARTHRITIS PATIENTS}

Ha-Reum Lee, Su-Jin Yoo, Jinhyun Kim, In Seol Yoo, Chan Keol Park, Seong Wook Kang. Chungnam National University College of Medicine, Department of Internal Medicine, Daejeon, Korea, Rep. of (South Korea)

Background: $T$ cells play a regulatory role in rheumatoid arthritis (RA) through inducing the homeostasis maintenance and self-tolerance ${ }^{[1]}$. Specially, the production and the oxidation mechanism of reactive oxygen species (ROS) were out of balance.

Objectives: The aim of the study was to compare ROS productions in $T$ cell subset, which are helper $\mathrm{T}(\mathrm{TH})$ cell, cytotoxic $\mathrm{T}$ (TC) cell, $\mathrm{T}$ helper 17 (TH17) cell and regulatory $\mathrm{T}$ (Treg) cell in peripheral blood mononuclear cells (PBMC) of RA patients with RA activity.

Methods: Blood samples were collected from 30 RA patients and 10 healthy adult volunteers under IRB approval. RA activity was divided according to clinical parameter DAS28 ${ }^{[2]}$. PBMC cells were obtained from the whole blood using lymphocyte separation medium density gradient centrifugation. For separation between the live and dead cell populations, PBMC was stained with Live/Dead stain dye. After PBS washing, cells were incubated with antibodies for CD3, CD4, CD8, and CD25. Following fixation and permeabilization, and further stained with antibodies for FoxP3 and IL-17A. For ROS staining, CellRox and MitoSox were used.
Results: The frequency of $\mathrm{TH}$ cell was increased and that of TC cell was decreased in the peripheral blood of RA patients. TH17 and Treg cell population were significantly increased more than about 2-3 folds in active and inactive RA than healthy control. When the whole of cellular ROS production was measured, only Treg cell population was significantly increased in RA than control. Although ROS level was steadily increased with RA activity, there was a slight decline in severe RA compared to moderate and low RA. This difference is lager in mitochondrial specific ROS than total cellular ROS. The mitochondrial complex inhibitor reduced Treg cell frequency in PBMC from RA patients.

Conclusion: Treg is the most sensitive to ROS production among $T$ cell subsets in RA. These findings provide a novel approach to regulate Treg function in RA through mitochondrial metabolism related ROS production.

\section{REFERENCES}

[1] Szekanecz, Z., et al., New insights in synovial angiogenesis. Joint Bone Spine, 2010. 77(1): p. 13-9.

[2] Prevoo, M.L., et al., Modified disease activity scores that include twentyeight-joint counts. Development and validation in a prospective longitudinal study of patients with rheumatoid arthritis. Arthritis Rheum, 1995. 38(1): p. 44-8.

Disclosure of Interests: None declared

DOI: 10.1136/annrheumdis-2019-eular.3850

\section{SAT0024 TRANSCRIPTOMIC PROFILING OF THE MICROENVIRONMENT DRIVEN RE-SHAPING OF PATHOGENIC CIRCULATORY AND SYNOVIAL HLA-DR+ CD4 T SUBSETS IN ACTIVE JUVENILE IDIOPATHIC ARTHRITIC PATIENTS}

Jing yao Leong ${ }^{1}$, Pavanish Kumar ${ }^{1}$, Phyllis Chen ${ }^{1}$, Joo Guan Yeo ${ }^{1}$,

Camillus Chua $^{1}$, Sharifah Nur Hazirah ${ }^{1}$, Thaschawee Arkachaisri ${ }^{1}, 2$,

Alessandro Consolaro ${ }^{3}$, Marco Gattorno ${ }^{3}$, Alberto Martini ${ }^{3}$, Salvatore Albani ${ }^{1}$.

${ }^{1}$ Translational Immunology Institute, Singhealth/Duke-NUS Academic Medical Centre, Singapore, Singapore; ${ }^{2}$ KK Women's and Children's Hospital, Singapore, Singapore; ${ }^{3}$ Second Paediatric Division, University of Genoa and G Gaslini Institute, Genova, Italy

Background: We have previously identified two pathogenic circulatory CD4 subsets in both Teff (CPLs) ${ }_{1}$ and Treg (iaTreg) $)^{2}$ compartments of JIA patients that are $\mathrm{HLA}-\mathrm{DR}^{+}$, antigen experienced, pro-inflammatory, correlating positively with disease activity and possessing strong synovial TCR sequence coverage. Despite being two functionally discordant $T$ cell subsets (Teff/Treg), their immuno-phenotype and association with clinical fate suggests that these subsets may originate from a common precursor.

Objectives: We seek to understand how the microenvironment could potentially influence and drive these subsets (CPLs/iaTregs) towards their pathological state. In an attempt to elucidate the common pathological gene drivers, we decided to perform next-generation RNA sequencing on sorted CPLs/iaTregs and conventional Teff/Treg counterparts in both circulation and synovium.

Methods: CPLs were sorted as $\mathrm{CD}^{+} \mathrm{CD}^{+} \mathrm{CD}^{+} 4^{-} \mathrm{HLADR}^{+} \mathrm{CD} 25 / \mathrm{CD} 127$ Teff gate, and iaTregs were sorted as $\mathrm{CD}^{+} \mathrm{CD}^{+} \mathrm{CD}^{-} 4^{-} \mathrm{HLADR}^{+}$ CD25 ${ }^{\text {hi }} / C D 127^{\text {low }}$ Treg gate with FACs Aria II from $n=16$ active JIA PBMCs, $n=8$ paired JIA SFMCs, and $n=8$ healthy paediatric PBMCs. As a comparative control, similar HLADR ${ }^{-}$counterparts were respectively sorted from the same patients. Sorted cells were lysed and extracted for RNA, and cDNA conversion/amplification were then carried out using SMART-seq v4. Libraries are prepared and multiplexed using Nextera XT DNA library preparation kit, and ran on the Illumina HiSeq High output platform.

Results: Comparative differential gene expression (DEG) analysis within the circulatory compartment indicate transcriptomic convergence between CPLs/iaTreg and divergence away from conventional Teff/Treg pools. Circulatory CPLs/iaTregs exhibit (a) common pathway dysregulation in $\mathrm{T}$ cell signalling (IFN-g, PD1, CD28 costimulation), (b) restriction in TCR oligoclonality and (c) common transcription factor drivers (SPL1 and E2F1) within the gene regulatory network, suggesting a common driving source acting on these two disparate compartments.

To understand how this convergence originate, we compared CPLs/iaTreg and conventional Teff/Treg subsets from (a) healthy circulatory PBMCs, (b) JIA circulatory PBMCs and (c) paired JIA synovium. There was a gradual increase in transcriptomic convergence between Teff/CPLs, Treg/ iaTreg and CPLs/iaTreg across the spatial/disease continuum, that is paralleled by an antigenic convergence in shared TCR clonotypes in CPLs/ 
iaTreg. In particular synovium CPLs/iaTreg reveal 7 common dysregulated pathways; MHC II antigen presentation, $\mathrm{T}$ cell costimulation, IFNg pathway, apoptosis, viral response, bacteria response and chemotaxis.

Conclusion: Overall the data indicate immune-phenotypic convergence between CPLs/iaTregs, that is strengthen across disease/spatial states. These findings underscore a potential mechanistic role of the inflammatory microenvironment in shaping two functionally dichotomic populations, relevant to disease pathogenies and progression.

\section{REFERENCES}

[1] Spreafico R, et al. A circulating reservoir of pathogenic-like CD4+ T cells shares a genetic and phenotypic signature with the inflamed synovial micro-environment. Ann Rheum Dis 75, 459-465 (2016).

[2] Rossetti M, et al. TCR repertoire sequencing identifies synovial Treg cell clonotypes in the bloodstream during active inflammation in human arthritis. Ann Rheum Dis 76, 435-441 (2017).

Disclosure of Interests: Jing yao Leong: None declared, Pavanish Kumar: None declared, Phyllis Chen: None declared, Joo Guan Yeo: None declared, Camillus Chua: None declared, Sharifah Nur Hazirah: None declared, Thaschawee Arkachaisri Speakers bureau: Abbvie Pte, Ltd, Alessandro Consolaro: None declared, Marco Gattorno Grant/research support from: MG has received unrestricted grants from Sobi and Novartis, Alberto Martini: None declared, Salvatore Albani: None declared DOI: 10.1136/annrheumdis-2019-eular.4464

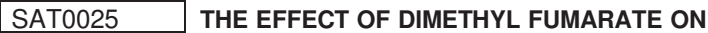 PLASMABLAST DIFFERENTIATION TRANSCRIPTIONAL PROGRAMMES IN SYSTEMIC LUPUS ERYTHEMATOSUS}

Daniele Mauro $^{1,2}$, Felice Rivellese ${ }^{1}$, Sotiria Manou-Stathopoulou ${ }^{1}$, Katriona Goldmann ${ }^{1}$, Debasish Pyne ${ }^{3}$, Francesco Ciccia ${ }^{4}$, Costantino Pitzalis ${ }^{1,3}$, Myles Lewis ${ }^{1,3}$. ${ }^{1}$ Queen Mary University of London - William Harvey Research Institute, Centre for Experimental Medicine and Rheumatology, London, United Kingdom; ${ }^{2}$ University of Palermo, Dipartimento biomedico di Medicina Interna e Specialistica Sezione di Reumatologia, Palermo, Italy, ${ }^{3}$ Mile End Hospital, Barts Health NHS Trust, Rheumatology Department, London, United Kingdom;

${ }^{4}$ University of Campania "Luigi Vanvitelli", Rheumatology, Naples, Italy

Background: Dimethyl fumarate (DMF), is an immunomodulatory drug approved for the treatment of Multiple Sclerosis (MS) and Psoriasis. The exact mechanism of action of DMF is not entirely known. Anti-inflammatory and immunomodulatory effects have been observed, including the upregulation of NRF-2, the inhibition of TIGAR and the block of the E2 ubiquitin-conjugating enzyme UBEL3. Further evidence from MS patients suggests a modulation on $B$ cell activation. Although beneficial effects of DMF have been observed in animal models of lupus nephritis and limited cases human cutaneous lupus, the effect of DMF on B cell maturation transcriptional programmes in systemic Lupus Erythematosus (SLE) has not been fully investigated.

Objectives: To examine the effect of DMF on SLE plasmablast differentiation and identify the transcriptomic changes in cultured SLE B cells after DMF administration.

Methods: B cells were isolated from the peripheral blood of SLE patients $(n=15)$ by negative magnetic sorting. B cell differentiation toward plasmablasts was induced in-vitro by stimulation with TLR-7 agonist Resiquimod, CD40L, IL-2, IL-10 and IL-15 for 5 days in the presence of DMF $25 \mathrm{uM}$ or vehicle, added $24 \mathrm{~h}$ after plating. IgG and $\lg \mathrm{M}$ production were quantified in the supernatant by ELISA. In-vitro differentiated B cells were immunophenotyped by 10 -colour flow cytometry in order to identify Naïve $\left(\mathrm{CD}^{-} 7^{-} \lg \mathrm{D}^{+}\right)$, Memory $\left(\mathrm{CD}^{2} 7^{+} \lg \mathrm{D}^{-}\right)$, $\lg ^{+}$Memory $\left(\mathrm{CD}^{+} 7^{+} \lg \mathrm{D}^{+}\right)$, Double Negative (DN) (CD27 lgD ${ }^{-}$) B cells, pre-plasmablasts (pre-PBs) $\left(\mathrm{CD}^{-} 7^{+}\right.$ CD20low, CD38 $)$ and plasmablasts (PBs) $\left(\mathrm{CD}^{-} 7^{+}, \mathrm{CD}^{\mathrm{low}}, \mathrm{CD}^{+} 8^{+}\right)$ Naïve $B$ cells and PBs from 3 patients were isolated by fluorescenceactivated cell sorting and underwent to RNA-sequencing followed by pathway analysis.

Results: DMF administered from day 2 led to a relative increase in the percentage of Naïve $B$ cells $(p=0.002)$ and a substantial reduction in Memory $B$ cells $(p=0.005)$, while the proportion of $D N$ cells and $\operatorname{lgD}^{+}$ Memory $\mathrm{B}$ cells were not altered. DMF reduced the percentage of PBs $(p=0.003)$, in contrast, the percentage of pre-PBs was significantly increased $(p=0.006)$. Consistently, $\lg G$ and $\lg M$ secretion were significantly reduced $(p<0.001)$. DMF treatment induced significant transcriptional changes in both PBs and Naïve B cells, with 269 and 652 genes modulated in PBs and Naïve B cells, respectively ( $\mathrm{Paj}<0.05)$. Pathway analysis highlighted the downregulation of a number of pathways: detoxification of reactive oxygen species, transcriptional regulation of cell cycle inhibitor p21, mitophagy and Pink/Parkin Mediated mitophagy, interleukin-12 signalling, TP53 and p53 regulation, PERK mediated endoplasmic unfolded protein response, and NF-kB mediated cell survival. Conversely, pathways related to epigenetic regulation, RUNX1 signalling, Rho and GTPase signalling were upregulated.

Conclusion: Our results show that Dimethyl Fumarate exerts a significant inhibition on SLE plasmablast differentiation and antibody production. The transcriptomic analysis allowed to dissect B cell transcriptional programmes activated in the context of autoimmune B cell differentiation in SLE. The transcriptional perturbations induced by DMF highlighted some of the gene expression pathways necessary for plasmablast differentiation and survival. In addition, these data provide new insight into DMF phar macodynamics and may support the repositioning of DMF in the treatment of SLE.

Disclosure of Interests: Daniele Mauro: None declared, Felice Rivellese: None declared, Sotiria Manou-Stathopoulou: None declared, Katriona Goldmann: None declared, Debasish Pyne: None declared, Francesco Ciccia Grant/research support from: CELGENE, PFIZER, Consultant for: UCB, NOVARTIS, CELGENE, PFIZER, LILLY, Paid instructor for: UCB, NOVARTIS, CELGENE, PFIZER, LILLY, JANSSEN, Speakers bureau: UCB, NOVARTIS, CELGENE, PFIZER, LILLY, JANSSEN, ROCHE, AMGEN, Costantino Pitzalis Grant/research support from: Celgene, Myles Lewis Grant/research support from: Celgene DOI: 10.1136/annrheumdis-2019-eular.7792

\section{SAT0026 IMPACT OF TOCILIZUMAB ON IMMUNE PHENOTYPES} IN PATIENTS WITH LARGE VESSEL VASCULITIS

Shingo Nakayamada, Yusuke Miyazaki, Hiroko Yoshinari, Akio Kawabe, Ippei Miyagawa, Satoshi Kubo, Shunsuke Fukuyo, Shigeru Iwata, Kazuhisa Nakano, Yoshiya Tanaka. University of Occupational and Environmental Health, Japan, School of Medicine, First Depertment of Internal Medicine, Kitakyushu, Japan

Background: The pathogenesis of large vessel vasculitis (LVV) such as Takayasu arteritis (TAK) and giant cell arteritis (GCA) consists of the immune abnormalities including the interaction between vascular dendritic cells, macrophages and $\mathrm{T}$ cells. It is reported that genetic polymorphisms in the immune-modulating cytokine genes such as IL6 and IL12B are associated with LVV. However, little is known about pathological immune cell subsets targeted by immunosuppressants and/or molecular-target therapy such as IL-6 blockade.

Objectives: The aim of this study was to assess the relationship between the phenotype of peripheral immune cells with clinical manifestations and responsiveness to the treatment in patients with LVV

Methods: Peripheral blood mononuclear cells were obtained from 22 patients with active LVV (TAK 7, GCA 15) and 19 healthy donors (HD). All patients were treated with high dose glucocorticoid (GC). The study included the patients treated with immunosuppressive agents such as azathioprine and methotrexate $(n=8)$ or with anti-IL-6 receptor antibody tocilizumab $(n=7)$. The blood samples were taken at baseline and week 24 after treatment. The peripheral immune cell subset was defined based on comprehensive 8-color flow cytometric analysis for human immune system termed "the Human Immunology Project" by $\mathrm{NIH}$ and FOCIS (1) and the correlation with clinical characteristics and responsiveness to the therapies were evaluated.

Results: The proportion of $\mathrm{CD}^{+} \mathrm{CD} 4^{+} \mathrm{CXCR} 3^{-} \mathrm{CCR} 6^{+} \mathrm{CD} 38^{+} \mathrm{HLA}-\mathrm{DR}{ }^{+}$activated Th17 cells and $\mathrm{CD}^{+}{ }^{+} \mathrm{CD} 4^{+} \mathrm{CXCR} 5^{+} \mathrm{ICOS}^{+} \mathrm{CD} 38^{+}$activated Tfh cells in patients with TAK and that of activated Th17 cells and CD19 ${ }^{+} \mathrm{CD}^{2} 0^{+} \mathrm{CD} 27^{-} \mathrm{lg} \mathrm{D}^{-}$double negative effector $\mathrm{B}$ cells in patients with GCA were significantly higher compared with HD. At baseline, the fre quency of activated Tfh cells showed positive correlation and that of $\mathrm{CD} 4^{+} \mathrm{CCR} 4^{+} \mathrm{CD} 25^{+} \mathrm{CD} 127^{\text {low }} \mathrm{T}$ regulatory (Treg) cells showed negative correlation with disease activity scores such as Indian Takayasu Activity Index (ITAS) $2010^{\text {(2) }}$ and ITAS.A (CRP) in both TAK and GCA. The immunosuppressive therapy improved the disease activity in all patients The frequency of activated Tfh cells was reduced by 24-week treatment with high dose GC and immunosuppressants in TAK and GCA. However the frequency of Th17 cells was not changed by those treatments. Of note, tocilizumab decreased the proportion of activated Th17 cells and increased the proportion of Treg cells in both TAK and GCA.

Conclusion: The results indicated that the abnormal $\mathrm{T}$ cell differentiation correlated with disease activity of LVV. Although Tfh cell activation was improved, Th17 cell activation was not changed by the conventional immunosuppressive agents. By contrast, tocilizumab reduced Th17 cells and increased Treg cells, indicating that IL-6 blockade may correct the impaired balance of Th17 and Treg cells in patients with LVV 